\title{
ON THE PHASE CROSSING STATISTICS AND RANDOM FM NOISE IN GENERALIZED RICE FADING CHANNELS
}

\author{
Marko D. Petković* — Mihajlo Č. Stefanović ${ }^{* *}$
}

\begin{abstract}
In this paper we consider phase process second order statistics of generalized Rice (Beckmann) multipath fading channel. Closed-form expression for JPDF of phase and random FM noise is derived. Furthermore expressions for the PDF and CDF of random FM noise are obtained. The level-crossing rate of the phase process is then obtained for any phase crossing level. Obtained expressions reduces to known ones for Hoyt, Rice and Rayleigh fading channels, since these are the special cases of generalized Rice fading channel. Moreover, derived analytical expressions are compared with results obtained by computer simulation where excellent agreement is achieved. Presented results can be applied for analyzing the statistics of FM spikes in the case of data transmission over generalized Rice fading channels.
\end{abstract}

K e y w o r d s: level crossing theory, Hoyt fading channels, generalized Rice fading channels, random frequency modulation (FM) noise, phase process

\section{INTRODUCTION}

In wireless communication, an accurate modeling of propagation channels is very important both for the system design and performances analysis. A large number models have been proposed to describe the statistics of the amplitude and phase of multipath fading signals in different scenarios and operating environments [1-4]. Rayleigh and Rice models are widely used for describing behavior of the small-scale multipath fading $[5,6]$. In these models in-phase and quadrature component of the signal are uncorrelated Gaussian processes having identical variances. Rice model generalizes Rayleigh model and is applicable when there exists LOS (Line Of Sight) signal component.

There are two ways for increasing the flexibility of these classical models in order to enable a better fit to the measurement data. The first way is considered in $[6,7]$ and assumes that Gaussian processes which describe the model are correlated. Other way is to assume that these processes have different variances. In such manner, a Hoyt (Nakagami- $q$ ) model [8] is regarded as the extension of Rayleigh model. It is independently described by Nakagami [9] as an approximation of Nakagami- $m$ model. It is shown $[5,11]$ that this model is applicable for describing the statistics of fading envelope in real-world mobile radio channels. Similar generalization of Rice model, known as generalized Rice model or Beckmann model, is introduced in [12]. Beckmann model assumes that multipath component is Hoyt faded and LOS component has constant amplitude and phase shift. In [13] it is shown that generalized Rice model fits accurately to measurement data on mobile satellite channels. First order statistics of this model are studied in $[14,15,16]$.
The level-crossing rate (LCR) and average fade duration (AFD) are very important quantities in the determination of the channel phase statistical properties. These quantities are known as second-order statistics and are extensively explored in the literature [3]. An expressions for LCR and AFD of envelope process for Hoyt and Beckmann fading model are given in [16] and [17] respectively. On the other hand, statistics of phase process and its derivative (also known as random frequency modulation (FM) noise) are important for some applications. An example of such application is the design of optimal carrier recovery schemes needed in the synchronization subsystem of coherent receivers [18]. Another notable example involves the performance of FM receivers using a limiterdiscriminator for detection, where random FM spikes generated by phase jumps deteriorate the error-rate performance [19]. Characterization of phase process and FM noise for Rayleigh and Rice models are given in, for example [2, 20, 3]. Similar work for Hoyt model is recently published in [17]. An intention of this paper is to generalize results from [17] and obtain the phase LCR and PDF of FM noise for generalized Rice model.

The remainder of the paper is organized as follows. In Section 2, the generalized Rice fading model is described and its first order statistics are reviewed. In Section 3 we derived PDF of random FM noise together with the JPDF of phase and FM noise. This JPDF is used in Section 4 where closed-form expression for phase LCR is obtained. In Section 5 we compared theoretical results with the computer simulation. Section 6 concludes the paper.

\footnotetext{
* University of Niš, Faculty of Sciences and Mathematics, Višegradska 33, 18000 Niš, Serbia, dexterofnis@gmail.com; ${ }^{* *}$ University of Niš, Faculty of Electronic Engineering, Aleksandra Medvedeva 14, 18000 Niš, Serbia, misa@elfak.ni.ac.rs
} 


\section{GENERALIZED RICE FADING MODEL AND FIRST ORDER STATISTICS}

The received signal in equivalent complex baseband, when transmitting an unmodulated carrier over generalized Rice fading channel $([13,16])$, is described by the Gaussian random process $\mu_{r}(t)$ defined by

$$
\mu_{r}(t)=A \exp \left(j \theta_{0}\right)+\mu_{1}(t)+j \mu_{2}(t)
$$

Here $\mu_{1}(t)$ and $\mu_{2}(t)$ are uncorrelated, zero mean, lowpass Gaussian processes with variances $\sigma_{1}^{2}$ and $\sigma_{2}^{2}$ respectively. Values $A$ and $\theta_{0}$ represents constant amplitude and phase shift of LOS component.

Envelope and phase processes, denoted by $R(t)$ and $\vartheta(t)$ respectively, can be obtained from (1) as modulus and argument of $\mu_{r}(t)$

$$
\begin{aligned}
R(t) & =\sqrt{\left(A \cos \theta_{0}+\mu_{1}(t)\right)^{2}+\left(A \sin \theta_{0}+\mu_{2}(t)\right)^{2}}, \\
\vartheta(t) & =\arctan \frac{A \sin \theta_{0}+\mu_{2}(t)}{A \cos \theta_{0}+\mu_{1}(t)} .
\end{aligned}
$$

First order statistics of generalized Rice fading process are investigated in $[13,16]$. Here we review these expressions. Since $\mu_{1}(t)$ and $\mu_{2}(t)$ are uncorrelated Gaussian processes, their JPDF (Joint Probability Density Function) is given by

$$
p_{X_{1}, X_{2}}\left(x_{1}, x_{2}\right)=\frac{1}{2 \pi \sigma_{1} \sigma_{2}} \exp \left(-\frac{x_{1}^{2}}{2 \sigma_{1}^{2}}-\frac{x_{2}^{2}}{2 \sigma_{2}^{2}}\right) .
$$

Let us introduce the transformation from cartesian to polar coordinates defined by:

$$
x_{1}+A \cos \theta_{0}=r \cos \theta, \quad x_{2}+A \sin \theta_{0}=r \sin \theta
$$

which Jacobian is $J=r$. By applying transformation (4) we obtain the following expression for the JPDF of envelope and phase process

$$
\begin{array}{r}
p_{R \vartheta}(r, \theta)=\frac{r}{2 \pi \sigma_{1} \sigma_{2}} \exp \left[-\frac{\left(r \cos \theta-A \cos \theta_{0}\right)^{2}}{2 \sigma_{1}^{2}}\right. \\
\left.-\frac{\left(r \sin \theta-A \sin \theta_{0}\right)^{2}}{2 \sigma_{2}^{2}}\right] .
\end{array}
$$

Integration of JPDF $p_{R \vartheta}(r, \theta)$ with respect to variable $\theta$ yields to

$$
p_{R}(r)=\frac{r}{2 \pi \sigma_{1} \sigma_{2}} \int_{0}^{2 \pi} \exp \left[-r^{2} h(\theta)+r A g\left(\theta_{0}, \theta\right)\right] \mathrm{d} \theta .
$$

Two auxillary functions $h(\theta)$ and $g\left(\theta_{0}, \theta\right)$ are defined as follows [16]

$$
\begin{aligned}
h(\theta) & =\frac{\cos ^{2} \theta}{2 \sigma_{1}^{2}}+\frac{\sin ^{2} \theta}{2 \sigma_{2}^{2}} \\
g\left(\theta_{0}, \theta\right) & =\frac{\cos \theta_{0} \cos \theta}{\sigma_{1}^{2}}+\frac{\sin \theta_{0} \sin \theta}{\sigma_{2}^{2}} .
\end{aligned}
$$

Similarly by performing an integration of (1) with respect to variable $r$ we derive the PDF of phase process:

$$
\begin{aligned}
& p_{\vartheta}(\theta)=\frac{1}{4 \pi \sigma_{1} \sigma_{2} h(\theta)} \exp \left[A^{2}\left(\frac{g^{2}\left(\theta_{0}, \theta\right)}{4 h(\theta)}-h\left(\theta_{0}\right)\right)\right] \times \\
& {\left[\frac{\pi}{2} \exp \left(-\frac{A^{2} g^{2}\left(\theta_{0}, \theta\right)}{4 h(\theta)}\right)-\frac{A g\left(\theta_{0}, \theta\right)}{2 \sqrt{h(\theta)}}\left(1-\operatorname{erf} \frac{A g\left(\theta_{0}, \theta\right)}{2 \sqrt{h(\theta)}}\right)\right] .}
\end{aligned}
$$

Here $\operatorname{erf}(x)$ is the error function defined by $\operatorname{erf}(x)=$ $\frac{2}{\sqrt{\pi}} \int_{0}^{x} \exp \left(-t^{2}\right) \mathrm{d} t$. It can be verified that (8) reduces to the known expressions for Rice and Hoyt fading [21] in cases $\sigma_{1}=\sigma_{2}=\sigma, \beta_{1}=\beta_{2}=\beta$ and $A=0$ respectively.

\section{STATISTICS OF RANDOM FM NOISE}

In this section we obtain the closed-form expression for the JPDF of phase process and its derivative $p_{\vartheta \dot{\vartheta}}(\theta, \dot{\theta})$. From this result, the PDF of phase derivative $\dot{\vartheta}(t)$ (Overdot will denote time derivative, $d / d t$ ) can be directly derived. Phase derivative process is also known as random FM noise $[17,18]$.

We start from the JPDF of $\mu_{1}(t), \mu_{2}(t), \dot{\mu}_{1}(t)$ and $\dot{\mu}_{2}(t)$ at the same time $t$. In the case when processes $\mu_{1}(t)$ and $\mu_{2}(t)$ has symmetrical Doppler PSD (Power Spectral Density) $[16,17,21]$ corresponding JPDF is given by the following expression

$$
\begin{aligned}
& p_{X_{1}, X_{2}, \dot{X}_{1}, \dot{X}_{2}}\left(x_{1}, x_{2}, \dot{x}_{1}, \dot{x}_{2}\right)= \\
& \quad \frac{1}{4 \pi^{2} \sigma_{1} \sigma_{2} \sqrt{\beta_{1} \beta_{2}}} \exp \left(-\frac{x_{1}^{2}}{2 \sigma_{1}^{2}}-\frac{x_{2}^{2}}{2 \sigma_{2}^{2}}-\frac{\dot{x}_{1}^{2}}{2 \beta_{1}}-\frac{\dot{x}_{2}^{2}}{2 \beta_{2}}\right),
\end{aligned}
$$

where $\beta_{1}$ and $\beta_{2}$ are variances of the processes $\dot{\mu}_{1}(t)$ and $\dot{\mu}_{2}(t)$ respectively. Note that in such case, all processes $\mu_{1}(t), \mu_{2}(t), \dot{\mu}_{1}(t)$ and $\dot{\mu}_{2}(t)$ are pairwise uncorrelated [3]. For the classical Jakes Doppler PSD [3], variances $\beta_{i}$ can be written as $\beta_{i}=2\left(\pi \sigma_{i} f_{\text {max }_{i}}\right)$ where $f_{\max _{i}}$ is maximal Doppler frequency of the process $\mu_{i}(t)$, for $i=1,2$. We assume that Gaussian processes $\mu_{1}(t)$ and $\mu_{2}(t)$ have different maximum Doppler frequencies, i.e. that $f_{\max _{1}} \neq$ $f_{\text {max }_{2}}$. Although this assumption lack of a clear physical basis, it allows to increase the flexibility of the model and therefore enables a better fitting to the measurement data.

For the purpose of deriving the PDF of random FM noise and the analysis of the crossing statistics, a JPDF $p_{R \dot{R} \vartheta \dot{\vartheta}}(r, \dot{r}, \theta, \dot{\theta})$ of processes $R(t), \dot{R}(t), \vartheta(t)$ and $\dot{\vartheta}(t)$ is required. This JPDF can be obtained by applying the transformation of cartesian coordinates $\left(x_{1}, x_{2}, \dot{x}_{1}, \dot{x}_{2}\right)$ to polar coordinates $(r, \theta, \dot{r}, \dot{\theta})$ given by the following relations

$$
\begin{aligned}
& x_{1}=r \cos \theta-A \cos \theta_{0}, \quad x_{2}=r \sin \theta-A \sin \theta_{0}, \\
& \dot{x}_{1}=\dot{r} \cos \theta-r \dot{\theta} \sin \theta, \quad \dot{x}_{2}=\dot{r} \sin \theta+r \dot{\theta} \cos \theta,
\end{aligned}
$$




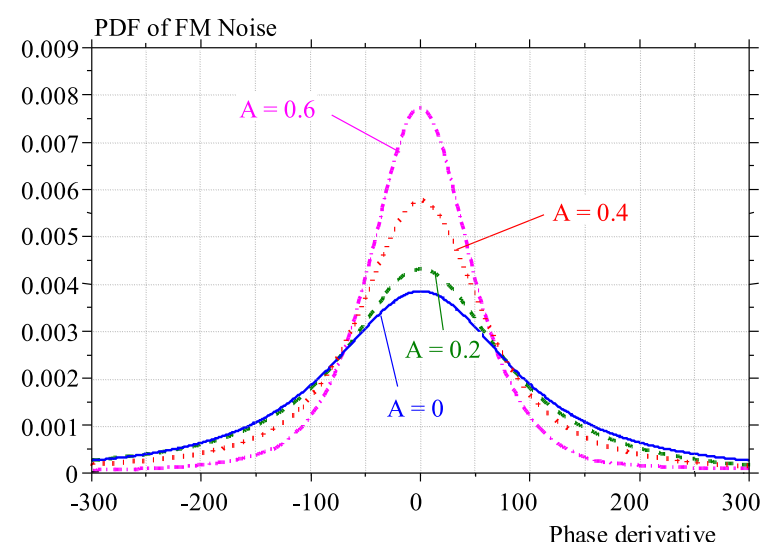

Fig. 1. PDF $p_{\dot{\vartheta}}(\dot{\theta})$ versus $\theta$ for different values of $A$ and $\theta_{0}=0$

and whose Jacobian is $J=r^{2}$. Combining (9) and relations (10) yields to the desired JPDF of processes $R(t), \dot{R}(t), \vartheta(t)$ and $\dot{\vartheta}(t)$

$$
\begin{aligned}
p_{R \dot{R} \vartheta \dot{\vartheta}}(r, \dot{r}, \theta, \dot{\theta})=\frac{r^{2}}{4 \pi^{2} \sigma_{1} \sigma_{2} \sqrt{\beta_{1} \beta_{2}}} \times \\
\exp \left[-\frac{\left(r \cos \theta-A \cos \theta_{0}\right)^{2}}{\sigma_{1}^{2}}-\frac{\left(r \sin \theta-A \sin \theta_{0}\right)^{2}}{\sigma_{2}^{2}}\right. \\
\left.-\frac{(\dot{r} \cos \theta-r \dot{\theta} \sin \theta)^{2}}{\beta_{1}}-\frac{(\dot{r} \sin \theta+r \dot{\theta} \cos \theta}{\beta_{2}}\right]
\end{aligned}
$$

where $r \geq 0,-\infty<\dot{r}<+\infty,-\pi<\theta<\pi$ and $-\infty<\dot{\theta}<+\infty$. This expression is the same as one in [16], but given in the different form.

Integration of (11) with respect to the variable $\dot{r}$ yields to the JPDF of $R(t), \vartheta(t)$ and $\dot{\vartheta}(t)$. In such manner we obtain

$$
\begin{aligned}
& p_{R \vartheta \dot{\vartheta}}(r, \theta, \dot{\theta})=\frac{r^{2}}{(2 \pi)^{3 / 2} \sigma_{1} \sigma_{2} \sqrt{\beta(\theta)}} \times \\
& \quad \exp \left[-A^{2} h\left(\theta_{0}\right)+r A g\left(\theta_{0}, \theta\right)-\frac{r^{2}}{2}\left(2 h(\theta)+\frac{\dot{\theta}^{2}}{\beta(\theta)}\right)\right] .
\end{aligned}
$$

Here $\beta(\theta)$ denotes the following auxillary function

$$
\beta(\theta)=\beta_{1} \sin ^{2} \theta+\beta_{2} \cos ^{2} \theta .
$$

Further integration of (12) with respect to $r$ yields to the required JPDF of phase and FM noise given by

$$
\begin{array}{r}
p_{\vartheta \dot{\vartheta}}(\theta, \dot{\theta})=\frac{1}{4(\pi a(\theta, \dot{\theta}))^{3 / 2} \sigma_{1} \sigma_{2} \sqrt{\beta(\theta)}} f\left(\frac{A g\left(\theta_{0}, \theta\right)}{\sqrt{2 a(\theta, \dot{\theta})}}\right) \times \\
\exp \left(-A^{2} h\left(\theta_{0}\right)\right) .
\end{array}
$$

where

$$
\begin{aligned}
f(s) & =2 s+\sqrt{\pi}\left(1+2 s^{2}\right) \exp \left(s^{2}\right)(1+\operatorname{erf}(s)), \\
a(\theta, \dot{\theta}) & =\frac{\dot{\theta}^{2}}{2 \beta(\theta)}+2 h(\theta) .
\end{aligned}
$$

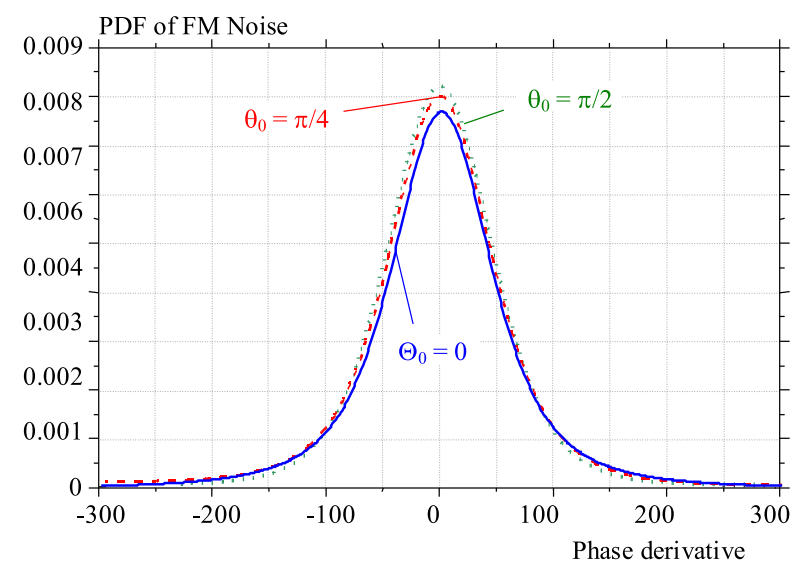

Fig. 2. $\operatorname{PDF} p_{\dot{\vartheta}}(\dot{\theta})$ versus $\theta$ for different values of $\theta_{0}$ and $A=0.6$

Note that in the case of Hoyt fading $(A=0)$ and Rician $\left(\sigma_{1}=\sigma_{2}=\sigma\right.$ ) expression (14) reduces to the corresponding expressions given in [3] and [17] respectively.

Consider the PDF of FM noise given by

$$
p_{\dot{\vartheta}}(\dot{\theta})=\int_{0}^{2 \pi} p_{\vartheta \dot{\vartheta}}(\theta, \dot{\theta}) \mathrm{d} \theta .
$$

Unfortunately, it seems that the last integral cannot be solved in the closed-form. Similarly we have CDF of random FM noise $F_{\dot{\vartheta}}(\dot{\theta})$ given by

$$
F_{\dot{\vartheta}}(x)=\int_{-\infty}^{x} \int_{0}^{2 \pi} p_{\vartheta \dot{\vartheta}}(\theta, \dot{\theta}) d \theta d \dot{\theta} .
$$

Note that integral in expression (17) also cannot be solved in closed-form. Nevertheless, we can study some properties of $p_{\dot{\vartheta}}(\dot{\theta})$. Similarly as for the Hoyt fading [17] holds that $\vartheta(t)$ has zero mean, ie $\mathbf{E} \dot{\vartheta}(t)=0$. This fact holds since $p_{\dot{\vartheta}}(\dot{\theta})$ is an even function of $\dot{\theta}$ (since $p_{\vartheta \dot{\vartheta}}(\theta, \dot{\theta})$ is an even function of argument $\dot{\theta}$ for each value $\theta \in[0,2 \pi))$. Moreover, the second moment of $p_{\dot{\vartheta}}(\dot{\theta})$ is infinite and therefore the variance $\sigma_{\dot{\vartheta}}^{2}$ is also infinite.

By numerical evaluation it can be found that $p_{\vartheta \dot{\vartheta}}(\theta, \dot{\theta})$ $\neq p_{\vartheta}(\theta) p_{\dot{\vartheta}}(\dot{\theta})$ and therefore phase process and FM noise are not statistically independent. It can be checked that such result is valid, also for the Hoyt fading (this is not yet reported in literature). In contract of that, for example, in the case of Rayleigh fading [3] phase and FM noise are statistically independent.

The PDF of random FM noise $p_{\dot{\vartheta}}(\dot{\theta})$ is evaluated for the parameter values $s_{1}^{2}=0.10391, s_{2}^{2}=0.030488, \beta_{1}=$ 1103.4298 and $\beta_{2}=1091.5206$. These values are given in [11] and are obtained by fitting the first and second order statistics of envelope $R(t)$ of Hoyt model to measurement data of an equivalent mobile satellite channel. Remember that generalized Rice model reduces to Hoyt by taking $A=0$.

Figures 1 and 2 show the graph of $p_{\vartheta}(\theta)$ for different values of LOS component amplitude $A$ and phase $\theta_{0}$ 


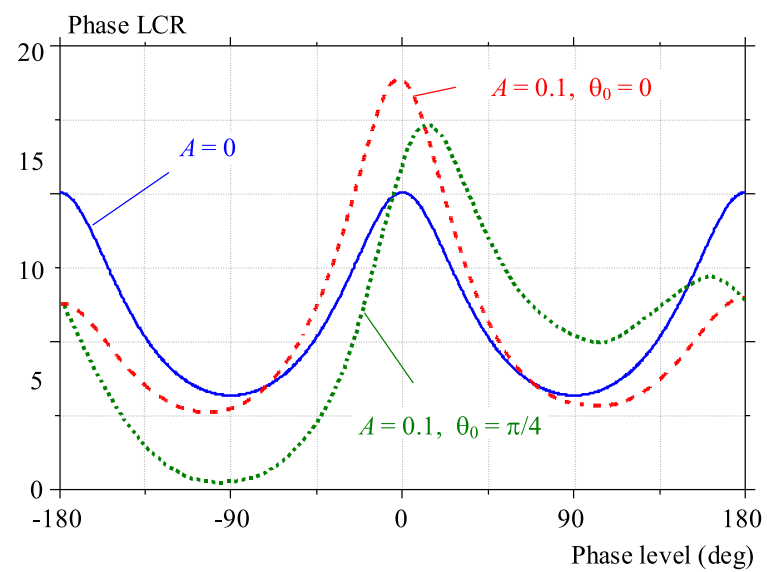

Fig. 3. Level-crossing rate $N_{\vartheta}^{+}(\theta)$ of the phase process $\vartheta(t)$ versus phase-crossing level $\theta$ for different values $A$ and $\theta_{0}$

respectively. As it can be seen $A$ has more significant influence on $p_{\vartheta}(\theta)$ than $\theta_{0}$. Graphs for $\theta_{0}=3 \pi / 4$ and $\theta_{0}=\pi$ are the same as graphs for $\theta_{0}=\pi / 4$ and $\theta_{0}=0$. This holds due to the fact that $g\left(\pi-\theta_{0}, \theta\right)=g\left(\theta_{0}, \theta\right)$ and $h\left(\pi-\theta_{0}\right)=h\left(\theta_{0}\right)$ and therefore values of $p_{\vartheta}(\theta)$ for $\theta_{0}=x$ and $\theta_{0}=\pi-x$ are equal.

\section{PHASE LEVEL-CROSSING RATE}

Level crossing rate (LCR) of the given process $X(t)$ is the expected number of times process crosses the specified threshold $x_{0}$ with positive slope. It is usually denoted by $N_{X}^{+}\left(x_{0}\right)$ and can be determined using the following expression [3]

$$
N_{X}^{+}\left(x_{0}\right)=\int_{0}^{+\infty} \dot{x} p_{X \dot{X}}\left(x_{0}, \dot{x}\right) d \dot{x} .
$$

Closed-form expressions for LCR of the envelope $R(t)$ are given in [16]. Here we deal with the phase process $\vartheta(t)$ and obtain its LCR (also known as PCR, Phase Crossing Rate) as a closed-form expression.

By performing an integration we obtain

$$
N_{\vartheta}^{+}(\theta)=\frac{\sqrt{\beta(\theta)}}{4 \pi \sigma_{1} \sigma_{2} \sqrt{h(\theta)}} \exp \left(-A^{2} h(\theta)\right) f_{1}\left(\frac{A g\left(\theta_{0}, \theta\right)}{2 \sqrt{h(\theta)}}\right) .
$$

Here $f_{1}(s)$ denotes the following auxillary function

$$
f_{1}(s)=\exp \left(s^{2}\right)(1+\operatorname{erf}(s))
$$

In the case $A=0$ (Hoyt fading) expression (19) becomes $N_{\vartheta}^{+}(\theta)=\sqrt{\beta(\theta)} /\left(4 \pi \sigma_{1} \sigma_{2} \sqrt{h(\theta)}\right)$, which is the same as expression (10) i [17]. In the case $\sigma_{1}=$ $\sigma_{2}=\sigma$ and $\beta_{1}=\beta_{2}=\beta$ (Rician fading), phase LCR (19) reduces to

$$
\begin{aligned}
N_{\vartheta}^{+}(\theta)=\frac{\sqrt{\beta}}{4 \pi \sigma} \exp \left(-\frac{A^{2} \sin ^{2}\left(\theta-\theta_{0}\right)}{2 \sigma^{2}}\right) \times & \\
& {\left[1+\operatorname{erf} \frac{A \cos \left(\theta-\theta_{0}\right)}{\sigma \sqrt{2}}\right] . }
\end{aligned}
$$

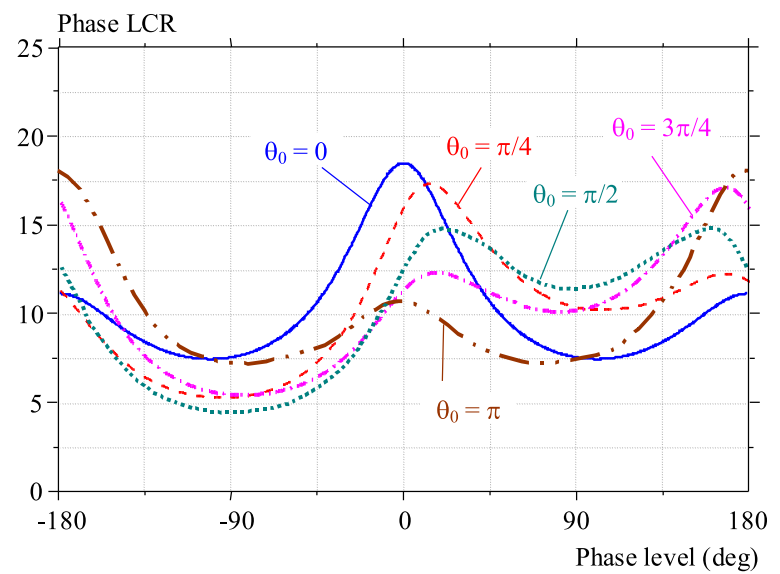

Fig. 4. Influence of LOS component phase $\theta_{0}$ on phase levelcrossing rate $N_{\vartheta}^{+}(\theta)$ for constant value $A=0.1$

If additionally holds $A=0$ (Rayleigh fading) then we have $N_{\vartheta}^{+}(\theta)=\gamma /(4 \pi)$. Here $\gamma=\sqrt{\beta} / \sigma$ is so-called radius of gyration of Doppler PSD of $\mu_{1}(t)$ and $\mu_{2}(t)$. Note that, in this case, PDF $p_{\vartheta}(\theta)=1 /(2 \pi)$ is constant and therefore $N_{\vartheta}^{+}(\theta)$ does not depend on phase level $\theta$. This is not true in the case $A \neq 0$ where $N_{\vartheta}^{+}(\theta)$ depends on $\theta$. It is worth mentioning that $N_{\vartheta}^{+}(\pi)$ is average number of $2 \pi$ positive noise spikes that could be observed at the frequency detector. The mean time interval, separating two consecutive noise spikes is given by $\bar{\tau}=2 \pi / N_{\vartheta}^{+}(\pi)$. By replacing $\theta=\pi$ in (19) we obtain

$$
\begin{aligned}
N_{\vartheta}^{+}(\pi)=\frac{\sqrt{\beta_{2}}}{4 \pi \sigma_{2}} \exp \left(-\frac{A^{2} \sin ^{2} \theta_{0}}{2 \sigma_{2}^{2}}\right) \times & \\
& {\left[1-\operatorname{erf} \frac{A \cos \theta_{0}}{\sigma_{1} \sqrt{2}}\right] . }
\end{aligned}
$$

Observe that (22) has the similar form as LCR for Rice fading model given by (21). In the case of Hoyt fading model, $N_{\vartheta}^{+}(\pi)$ has the same form as LCR for the Rayleigh fading [17].

In figures 3 and 4 , a graph of $N_{\vartheta}^{+}(\theta)$ versus crossing level $\theta$ is shown. We also used values of the parameters $\sigma_{1}, \sigma_{2}, \beta_{1}$ and $\beta_{2}$ from [11].

In figure 3 we compared three cases

- case of Hoyt fading ( $A$ is zero),

- case when phase of LOS component $\theta_{0}$ is zero, but $A$ is not zero,

- case when both envelope and phase of LOS component are not zero.

It can be seen that in first two cases $(A=0$, and $A \neq 0$ but $\theta_{0}=0$ ) phase level-crossing rate $N_{\vartheta}^{+}(\theta)$ is an even function of argument $\theta$. This is not true in the third case. These facts can be directly obtained from (19).

Consider now the case when maximum Doppler frequencies $f_{\max _{1}}$ and $f_{\text {max }_{2}}$ are equal. Let $f_{\max _{1}}=$ $f_{\text {max }_{2}}=f_{\text {max }}$. Then holds $\beta(\theta)=2\left(\pi f_{\text {max }} \sigma_{1} \sigma_{2}\right)^{2} h(\theta)$. 


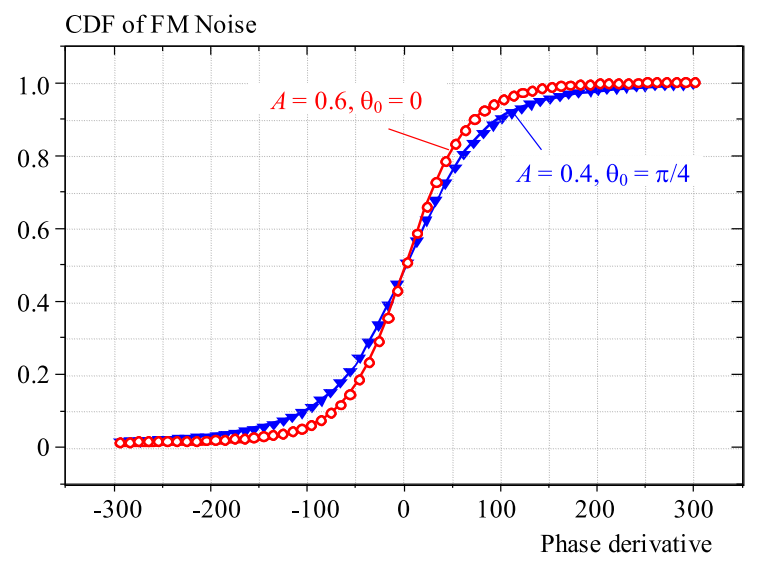

Fig. 5. CDF $F_{\dot{\vartheta}}(\dot{\theta})$ versus $\theta$ in two different cases. Comparison between theory - expression (17)- and results obtained by simulation

By replacing in (19) we obtain

$$
N_{\vartheta}^{+}(\theta)=\frac{f_{\max }}{2 \sqrt{2}} \exp \left(-A^{2} h(\theta)\right) f_{1}\left(\frac{A g\left(\theta_{0}, \theta\right)}{2 \sqrt{h(\theta)}}\right) .
$$

Let us mention that in the case $A=0$ (Hoyt fading) we have $N_{\vartheta}^{+}(\theta)=f_{\max } /(2 \sqrt{2})$ which is independent of crossing level $\theta$ (as it is reported in [17]). In general case when $A \neq 0$ such conclusion does not hold.

Figure 4 shows the dependence of $N_{\vartheta}^{+}(\theta)$ versus LOS component phase $\theta_{0}$. From (19) it is evident that $N_{\vartheta}^{+}\left(\theta_{0}\right)=N_{\vartheta}^{+}\left(2 \pi-\theta_{0}\right)$. Therefore it is sufficient to consider values $\theta_{0} \in[0, \pi]$. It can be seen that all curves, except one for $\theta=0$ has two maxima between $\pi / 2$ and $\pi$.

\section{SIMULATION}

For the conformation of obtained theoretical results for FM noise statistics and phase LCR, a computer simulation is performed. A deterministic method, based on the Rice's sum of sinusoids and described in [21,22] is used. Recall that in this model, Gaussian processes $\mu_{i}(t)$ $(i=1,2)$ are approximated by the following sum of cosine waves

$$
\tilde{\mu}_{i}(t)=\sum_{n=1}^{N_{i}} c_{i, n} \cos \left(2 \pi f_{i, n}+\theta_{i, n}\right)
$$

Values $c_{i, n}, f_{i, n}$ and $\theta_{i, n}$ are called gains, discrete Doppler frequencies and phases respectively and $N_{i}$ denotes the number of sinusoids used in simulation. There are several methods for computation of these quantities. We used Method of Exact Doppler Spread (MEDS) described in [22]. According to MEDS, simulation parameters are given by $c_{i, n}=\sigma_{i} \sqrt{2 / N_{i}}, f_{i, n}=f_{\max _{i}} \sin \left[\left(\pi /\left(2 N_{i}\right)\right)(n-\right.$ $1 / 2)$ ] and $\theta_{i, n}$ are realizations of uniform distributed random variable on interval $[0,2 \pi)$. Note that maximum

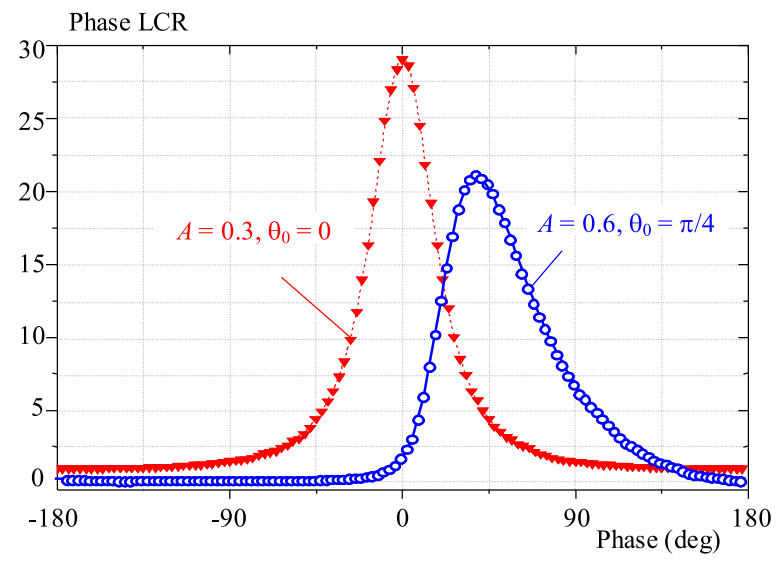

Fig. 6. Level-crossing rate $N_{\vartheta}^{+}(\theta)$ of the phase process $\vartheta(t)$ versus phase-crossing level $\theta$ in two different cases. Comparison between theory (expression (19)) results obtained by simulation

Doppler frequencies can obtained from relation $\beta_{i}=$ $2\left(\pi \sigma_{i} f_{\text {max }_{i}}\right)^{2}$.

For the simulation purpose, we used the same values of input parameters $s_{1}^{2}=0.10391, s_{2}^{2}=0.030488, \beta_{1}=$ 1103.4298 and $\beta_{2}=1091.5206$. We compared the values of CDF $F_{\dot{\vartheta}}(\dot{\theta})$ and phase LCR $N_{\vartheta}^{+}(\theta)$ obtained by theory (expressions (17) and (19)) and by simulation. Results are shown in figures 5 and 6 . As it can be seen, there is excellent agreement between theory and simulation in both figures.

\section{CONCLUSION}

In this paper, we studied the phase level-crossing rate (LCR) and statistics of random FM noise in generalized Rice (Beckmann) fading channels. The JPDF of phase process and its time derivative (random FM noise) is obtained in closed-form. This leads to the expression for PDF and CDF of random FM noise. Phase level-crossing rate is also obtained in the closed-form. All expressions are considered in special cases of Hoyt, Rice and Rayleigh channels and agreement with known expressions for this cases is shown. In order to verify obtained expressions, a computer simulation is performed. The derived analytical expressions are found to be in excellent agreement with those obtained by computer simulations.

All presented results can be applied for analyzing the statistics of FM spikes in the case of data transmission over generalized Rice fading channels.

\section{REFERENCES}

[1] ABDI, A.-LAU, W. C.-ALOUINI, M. S.-KAVEH, M. : A New Simple Model for Land Mobile Satellite Channels: First And Second Order Statistics, IEEE Trans. Wireless Commun. 2 No. 3 (May 2003 ), 519-528.

[2] HASHEMI, H.: The indoor radio propagation channel, Proc. IEEE 81 No. 7 (July 1993 ), 943-968 .

[3] JAKES, W. C. Ed. : Microwave Mobile Communications, Wiley, NJ, 1997. 
[4] Simon, M. K.-AlOUINI, M. S.: A unified Approach to the Performance Analysis of Digital Communication over Generalized Fading Channels, Proc. IEEE 86 No. 5 (Sep 1998 ), 1860-1877.

[5] PARSONS, J. D.: The Mobile Radio Propagation Channel, Pentech Press, London, 1992 .

[6] PAETZOLD, M.: Mobile Fading Channels, Wiley, New York, 2002.

[7] KRANTZIK, A.-WOLF, D. : Distribution of the Fading Time Intervals of Modified Suzuki Process, Signal Processing V: Theory and Applications (1990), 361-364.

[8] HOYT, R. S. : Probability Functions for the Modulus and Angle of the Normal Complex Variate, Bell Syst. Tech. J. 26 (1947), 318-359.

NAKAGAMI, M. : The $m$-Distribution - A General Formula of Intensity Distribution of Rapid Fading, Statistical Methods in Radio Wave Propagation, Pergamon, New York, 1960 , pp. 3-36,

[10] MEHRIA, A.-HASHEMI, H.: Mobile Satellite Propagation Channel Part II - A New Model and its Performance, in Proc. VTC99, Amsterdam,, Sep 1999 , pp. 2780-2784.

[11] WANG, C. X.-YOUSSEF, N.-PAETZOLD, M.: LevelCrossing Rate and Average Duration of Fades of Deterministic Simulation Models for Nakagami-Hoyt Fading Channels, in Proc. WPMC02, Honolulu, HI, Oct 2002 , pp. 272-276 .

[12] BECKMANN, P.-SPIZZICHINO, A.: The Scattering of Electromagnetic Waves from Rough Surfaces, Artech, Norwood MA, 1987 .

[13] XIE, Y.-FANG, Y.: A General Statistical Channel Model for Mobile Satellite System, IEEE Trans. Veh. Technol. 49 No. 3 (May 2000 ), 744-752.

[14] YACOUB, M. D.-FRAIDENRAICH, G.-TERCIUS, H. B.MARTINS, F. C. : The Symmetrical Eta-Kappa Distribution: a General Fading Distribution, IEEE Trans. on Broadcasting 51 No. 4 (Dec 2005 ), 504-511.

[15] YACOUB, M. D.-FRAIDENRAICH, G.-TERCIUS, H. B.MARTINS, F. C. : The Asymmetrical Eta-Kappa Distribution: a General Fading Distribution, IEEE Journal of Communication and Information Systems, 20 No. 3 (Dec 2005 ), 182-187.

[16] YOUSSEF, N.-WANG, C. X.-PAETZOLD, M.-JAAFAR, I.-TABANE, S.: On the Statistical Properties of Generalized Rice Multipath Fading Channels, VTC'04, Italy, vol. 1, 2004 , pp. $162-165$.

[17] YOUSSEF, N.-ELBAHRI, W.-PATZOLD, M.-ELASAMI, S. : On the Crossing Statistics of Phase Processes and Random FM Noise in Nakagami-q Mobile Fading Channels, IEEE Trans. Wireless Commun. 4 No. 1 (Jan 2005 ), 24-29.
18] HESS, D. T. : Cycle Slipping in the First Order Phase Locked Loop, IEEE Trans. Commun. Technol. 16 (Apr 1968 ), 255-260.

19] VAUGHAN, R. G. : Signals in Mobile Communications: A Review, IEEE Trans. Veh. Technol. 35 (Nov 1986 ), 133-145.

20] DAVIS, A. R.: Random FM in Mobile Radio with Diversity, IEEE Trans. Commun. Technol. 19 (Dec 1971 ), 1259-1267.

[21] YOUSSEF, N.-WANG, C. X.-PAETZOLD, M. : A study on the Second Order Statistics of Nakagami-Hoyt Mobile Fading Channels, IEEE Trans. Veh. Technol. 54 No. 4 (July 2005 ), 1259-1265.

[22] PAETZOLD, M.-KILlAt, U.-LAUE, F.-LI, Y.: On the Statistical Properties of Deterministic Simulation Models for Mobile Fading Channels, IEEE Trans. Vehic. Technol. VT-47 (Feb 1998 ), 254-269.

Received 20 January 2011

Marko D. Petković was born in Niš, Serbia, in 1984. He graduated mathematics and computer science at the Faculty of Sciences and Mathematics, Niš, Serbia in 2006, and telecommunications at Faculty of Electronic Engineering, Niš, Serbia in 2007. He received $\mathrm{PhD}$ degree in computer science from University of Niš in 2008. Currently he works as assistant professor at the Faculty of Sciences and Mathematics, Niš, Serbia. His research interests include the source and channel coding, generalized inverses of matrices, Hankel determinants and optimization methods. He is the author of about 50 papers (30 of them in peer-reviewed international journals). Dr Petković has been Reviewer for Journal of Computational and Applied Mathematics, Computers and Mathematics With Applications and International Journal of Computer Mathematics. He is supported by Ministry of Science, Republic of Serbia, Grant No. 174013

Mihajlo Č. Stefanović was born in Niš, Serbia, in 1947. He received his $\mathrm{BSc}, \mathrm{MSc}$ and $\mathrm{PhD}$ degrees in electrical engineering from the Faculty of Electronic Engineering (Department of Telecommunications), University of Niš, Serbia, in 1971, 1976 and 1979, respectively. His primary research interests are statistical communication theory, optical and wireless communications. He has authored or co-authored a large number of journal publications. He has written five monographs. Now, Dr Stefanović is full professor at Faculty of Electronic Engineering in Niš. 